\title{
Claim-based versus network-based identity management: a hybrid approach
}

\author{
Faysal Boukayoua $^{1}$, Jan Vossaert ${ }^{1}$, Bart De Decker ${ }^{2}$, and Vincent Naessens ${ }^{1}$ \\ 1 Katholieke Hogeschool Sint-Lieven, Department of Industrial Engineering \\ Gebroeders Desmetstraat 1, 9000 Ghent, Belgium \\ firstname.lastname@kahosl.be \\ 2 Katholieke Universiteit Leuven, Department of Computer Science, \\ Celestijnenlaan 200A, 3001 Heverlee, Belgium \\ firstname. las tname@cs. kuleuven. be
}

\begin{abstract}
This paper proposes a hybrid approach that combines claimbased and network-based identity management. Partly by virtue of the principle of separation of concerns, better security and privacy properties are attained. Overall trust is diminished, while simultaneously reducing multiple actors' exposure and value as a target of attack. The proposed architecture also facilitates interoperability and pluralism of credential technologies, authentication protocols and operators. In addition, the user has more control over his personal data than with current network-based identity management systems. A prototype demonstrates the feasibility of the proposed approach.
\end{abstract}

Key words: privacy, security, claim-based identity management, networkbased identity management

\section{Introduction}

Several definitions exist for identity management. The term is defined as follows by [2: Identity management consists of the processes and all underlying technologies for the creation, management, and usage of digital identities. In practice, identity management involves the creation, maintenance, usage and destruction of an entity's identity, typically as an enabler to meet the needs of security and business applications [2]20.

Different taxonomies on identity management systems have been devised in the past [4]18. The relevant distinction for this paper is between network-based systems and their more user-centric, claim-based counterparts [2]. Both comprise three major actors: the user, the service provider and the identity provider. In a network-based system, the user is typically redirected to an identity provider, which authenticates the user and supplies a token upon successful authentication. This token is then verified by the service provider and access is granted if it is valid. The procedure also includes the provisioning of any required user attributes, from the identity provider to the service provider. In a claim-based system, on the other hand, the service provider informs the user about the prerequisites to fulfill. If the user consents, he returns a claim. The latter can be regarded as a response to the server's challenge, along with a verifiable statement about required user attributes. A claim may be endorsed by one or multiple issuers. The user sits in between the identity providers and the service provider, thereby controlling the information exchange between the 
two sides. This leads to less trust assumptions and better privacy properties in comparison to a typical network-based system, where the identity provider's role is more pervasive. The latter is also capable transaction monitoring and linking, collusion with other identity or service providers, and user impersonation. In addition, the concentration of multiple security-critical responsibilities makes network-based identity providers a high-value (single) point of attack. However, in terms of interoperability and standardisation, network-based systems have an advantage over their claim-based counterparts. For instance, deployment of eID technology is typically country-wide, thereby imposing additional efforts at best, or excluding international users altogether. Moreover, there is a need to facilitate the adoption of new privacyfriendly credential technologies 107622 . The reuse of existing infrastructure and expertise is important in this respect.

Contribution: This paper synthesises a hybrid counterpart of network-based and claim-based identity management architectures, striving to leverage strengths and mitigate weaknesses. The modifications result in a clear separation of concerns, which yields advantages in terms of trust, privacy, security and interoperability. The feasibility of the proposed approach is demonstrated by a Shibboleth-based implementation that incorporates Idemix and the claim-based research architecture described in 22 .

After elaborating on related work in section 2 an architecture is defined in 3 . The latter is then validated in section 4 by means of the implementation and evaluation of a prototype. The section also explores possible extensions and variants. Following an overall evaluation in 5 , conclusions are drawn in 6 .

\section{Related work}

The current identity management landscape is a diverse one. Among claim-based systems, we categorise data-minimising credential technologies (Idemix [10] and UProve [7]), government-deployed eID technology (e.g. the Belgian [12] and the German [6] eID) as well as conventional, X509 software certificates. The architecture described in [22] is claim-based as well. A number of claim-based systems offer interesting privacy and security properties. The German eID, for instance, requires a PIN entry before releasing personal attributes [5]. Moreover, it can generate service provider-specific transaction pseudonyms. Idemix and U-Prove, on the other hand, both provide pseudonymous and anonymous authentication and minimal attribute disclosure.

Popular network-based identity management systems include Shibboleth [17, which is widely used in educational institutions. OpenID [19 on the other hand, is used as an alternative to conventional username-password Web authentication. Proprietary infrastructures like Google's and Facebook's can also be listed here. The adoption of purely network-based systems is less hampered by the requirement for additional installations or configurations on the user's system, like many claimbased systems. Standardised specifications like SAML and ID-WSF are adopted and backed on an interorganisational level. Shibboleth, for instance, is used by major academic and research networks [24] [23].

Albeit each to its own extent, network-based systems have drawbacks. OpenID poses a wide range of security and privacy issues [8]. Moreover it lacks a mechanism 
for trust establishment (cf. Shibboleth federations). Network-based systems regularly have no support for selective attribute disclosure, although there are examples of the opposite. The concept is denoted as permission-based attribute sharing in the ID-WSF specification [3]. uApprove [14] is a plugin that enhances Shibboleth with similar functionality. These features and improvements contribute to privacy, compared to systems where they are not available. However network-based identity providers still have considerable power for transaction linking and monitoring, profiling, collusion with other identity providers or service providers. Lastly, they combine storage of user information and user authentication, 2 security-critical tasks.

Related research has been put forward by Dodson et al. [13. It proposes a phonebased out-of-band authentication to an OpenID provider using a symmetric keybased challenge-response protocol. The approach by [15] is similar, combining the German eID with OpenID. Apart from hindering phishing, both approaches also replace user names and passwords by strong authentication. However, the OpenID trust establishment issue remains unsolved. Moreover, as the OpenID provider still stores user attributes, it remains a high value attack target. The approach by Leicher et al. [16, on the other hand, integrates the infrastructure of a user's mobile phone operator into OpenID. Besides the improvements of the two previous works, this approach also introduces trust establishment in OpenID by relying on trust in the operator and on the tamperproofness of a SIM card. Furthermore, it extends OpenID with pseudonymity and anonymity. However, it mainly focuses on attaining better security, privacy and usability. Interchangeability of claim-based credential technologies and network-based authentication protocols fall beyond the scope of all three papers.

Additional related work is outlined in ABC4Trust [9. It elaborates, among others, on how to integrate claim-based Privacy-ABCs in existing network-based systems. Their architectural margin for extensions is explored, after which integration strategies are discussed. However, each network-based system is treated separately. This paper strives to generalise this to support many claim-based technologies and network-based protocols, leaving the eventual choice up to the user's preference.

\section{Architecture}

In this section, the architectural requirements are introduced. Subsequently, we discuss modifications to network-based systems to satisfy these requirements.

\subsection{Requirements}

\section{General requirements}

$G_{1}$ Security issues in the network-based authentication protocol, are resolved or mitigated. The specific problems and the extent to which they are treated, is specific to the network-based system that is considered.

$G_{2}$ Pluralism of operators, claim-based credential technologies, as well as networkbased authentication protocols, is facilitated [11].

\section{User requirements}

$U_{1}$ User trust in the network-based identity provider is decreased. Depending on the incorporated claim-based and network-based systems, this requirement ideally results in reduced capabilities for transaction linking and profiling. 
$U_{2}$ The user has control over which personal attributes are released.

$U_{3}$ Switching to a different claim-based technology, poses no inconvenience to the user.

It is technologically easy for the user to switch to a another claim-based credential technology.

\section{Service provider requirements}

$S P_{1}$ Service providers can obtain reliable user information for service personalisation.

$S P_{2}$ The service provider is agnostic towards credential technologies and authentication protocols: he needs to support only few implementations to reach the majority of users.

\subsection{Components}

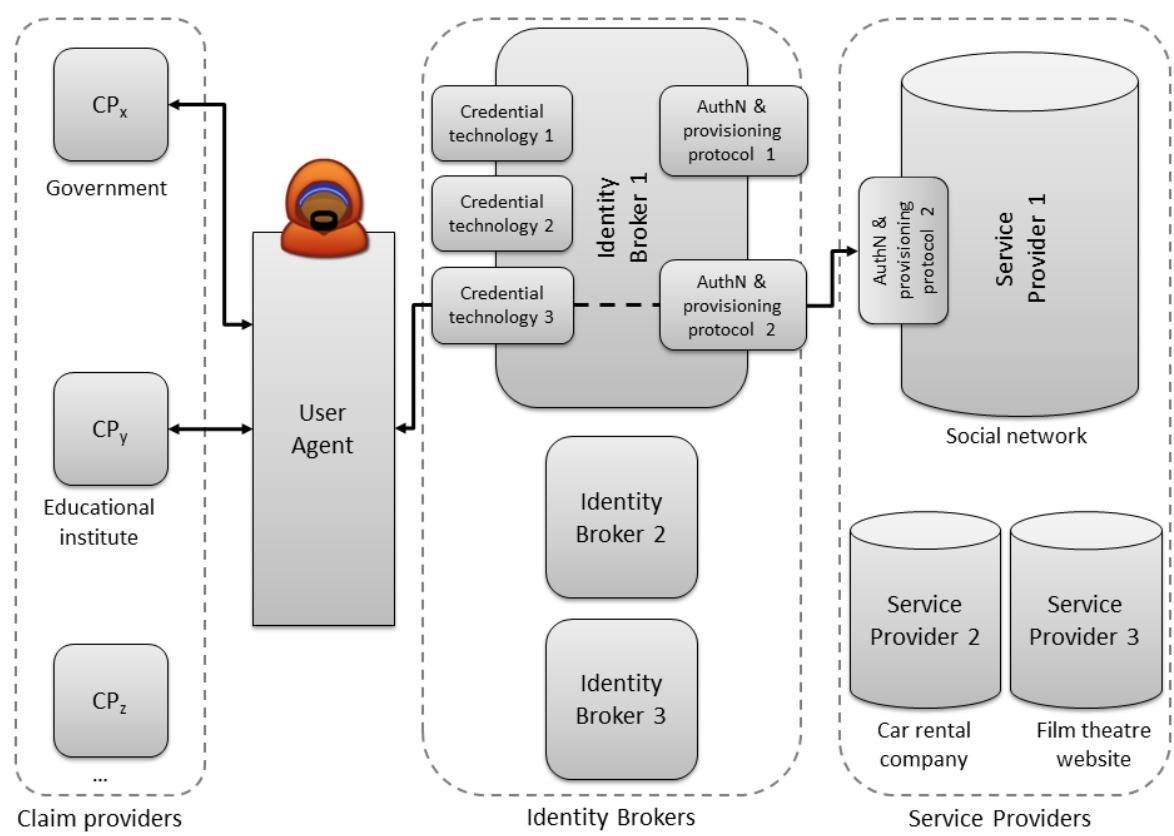

Fig. 1. The proposed identity management architecture.

The proposed architecture is depicted in figure 1. There are four components: the service provider, the identity broker, the user agent and the claim provider.

Service provider Service providers offer personalised services to users. To enable personalisation, the release of a set of user attributes is required during authentication. Service providers interact with an identity broker (see below) to authenticate users and obtain personal information from them. Hence, their role and implementation is unchanged, compared to the original network-based service providers. 
Identity broker Similar to claim-based systems, the user presents to the identity broker a claim proving the fulfillment of the service provider's requirements. However, in the proposed architecture, the identity broker extracts the user attributes or their properties, and provisions them to the service provider. This is performed according to a the network-based authentication protocol. Hence, brokers can be regarded as a conversion layer. They no longer store and maintain user information.

An identity broker implements credential-specific connectors to authenticate the user and obtain required user attributes. Each connector supports a credential technology. Once proven, the required attributes are transferred to service provider. This step can be achieved using any network-based authentication protocol that is supported by the identity broker: OpenID, SAML,...

Claim provider The claim-based identity provider, or claim provider for short, corresponds with the issuer of a claim-based system. It is henceforth the only responsible for user attribute storage and maintenance, whereas the network-based identity provider is transformed to an identity broker. Each claim provider can support a different technology.

User agent Similar to the ABC4Trust project terminology, the user agent is responsible for managing the user's claims. Multiple technologies may be incorporated. Some necessitate no online presence of the claim provider during authentication, while others may require the user agent to fetch additional credentials or attributes on the fly. This component is typically implemented on a (portable) device, like a smartphone or a smartcard.

Apart from managing credentials and authenticating the user to service providers, the user agent also implements other useful functionality [21]. This may include a connection component, providing support for different communication types: TCP/IPbased socket connections, HTTP, Bluetooth, etc. Furthermore, a privacy component can display the service provider the user is authenticating to, along with the personal attributes that are about to be released. In addition, the user agent allows the user to select the claims he wants to authenticate with. User preferences can be automated as policies. They comprise restrictions that can be posed on communication channels, credentials, attribute disclosure,... Lastly, the user agent can also give feedback about the anonymity impact of the ongoing authentication.

\subsection{Trust relationships}

This subsection shortly reflects on the changes in trust relationships.

User $\rightarrow$ identity broker: The identity broker attests the user's identity to the service provider. The user no longer needs to entrust the broker with the storage of his personal data, as this is now assigned to claim providers, of which the corresponding credential technology often provides better privacy properties purely network-based approaches. For instance, user profiling is far less trivial in an anonymous transaction where only a common attribute (e.g. gender $=$ "F") or a property thereof (e.g. age $>18$ ) needs to be attested.

User $\rightarrow$ claim provider: The user trusts the claim provider to securely store a justifiable subset of his personal data. Note that, the user has multiple partial 
identities that are distributedly stored across claim providers. Additionally, this actor is also trusted to only perform authenticated provisioning to the user agent. The claim provider cannot monitor or link the user's transactions.

Service provider $\rightarrow$ claim provider: The service provider trusts the claim provider to only enforce genuine user attributes.

Service provider $\rightarrow$ identity broker: The service provider trusts the identity broker to only assert verified attributes. Furthermore, the service provider may request from the identity broker to only accept credentials from a trusted set of claim providers. This allows the service provider to maintain a clear overview of trusted actors in the system.

\section{Validation}

A proof of concept of the architecture in section 3 is implemented using Shibboleth as a network-based identity management system. The Shibboleth service provider remains unchanged upon its integration in the proposed architecture. This implies that the network-based authentication protocol between the service provider and the identity broker is unaltered, compared to a standard Shibboleth configuration. The user agent is implemented on a smartphone for two major reasons. The device is nearly always in the user's presence and most often bound to the user exclusively. An additional advantage is the widespread adoption of smartphones. The user agent interacts with the identity broker. Modifications to Shibboleth to support this, consist of integrating authentication and provisioning support for the two claim-based credential technologies. The first claim provider is an Idemix issuer, whereas the second one is part of the identity management architecture described in [22]. Both systems allow for selective disclosure and support pseudonymous and anonymous transactions.

\subsection{Interactions}

Two distinct setups are implemented. In the first one, a service is consumed on the user's smartphone. In the second configuration, the Web service is accessed on a device other than the user agent (e.g. a workstation and a smartphone, respectively).

In both cases, the user's browser is typically redirected to the Shibboleth Discovery Service. Next, the user selects his preferred identity broker. The latter is responsible for the endorsement of the user's attributes. The next steps are discussed separately, due to the differences between both scenarios.

First setup: the device on which the service is consumed, also contains the user agent In the first setup, the user agent on the smartphone has direct access to the session context of the transaction that was initiated with the identity broker. The user agent can, hence, authenticate the user through this session. This is similar to a standard Shibboleth setup that implements advanced authentication mechanisms, such as PKI-based authentication.

Second setup: the device on which the service is consumed, does not contain the user agent This is a more complex setup, as the authentication and the service request do not share the same session context. Therefore, a binding needs to be established between the two. This is carried out as follows: 
1. the identity broker generates a QR barcode, containing a credential-specific authentication challenge. In addition, it also contains a policy that must be met for the authentication to succeed. For example, a policy may demand a proof that the user is over the age of 18 , and that the authentication response be sent back only over HTTP. The barcode also contains the ID of the session that is to be authenticated.

2. the barcode is displayed by the device on which the service is consumed (e.g. a workstation). The user subsequently scans the QR code with his the smartphone.

3. the user agent generates an authentication response and transfers it back to the identity broker, using a secure and authentic out-of-band communication channel.

4. The authentication response is verified. If valid, the identity broker extracts the user's personal attributes (or properties thereof) from the response and generates a SAML response, which is subsequently transferred to the service provider, using the standard Shibboleth protocol.

\subsection{Implementation details}

The prototype builds upon Shibboleth v2. The last paragraph of section 3.3 mentions the ability of the service provider to specify a trusted list of claim providers. The implementation at hand, attains this by incorporating this list in the Shibboleth SAML Metadata. The latter is exchanged when service providers and identity providers join a Shibboleth trust federation.

Furthermore, the user agent is implemented on an Android-powered smartphone, running Idemix 2.3 and the identity management architecture in $[22$.

Authentication Support for the two incorporated claim-based credential systems is added by implementing a Shibboleth LoginHandler for each credential technology. This component authenticates the user, also obtaining required personal attributes along the way. During this procedure, the identity broker transfers information about the service provider to the user agent. This allows the latter to inform the user about the authentication details.

Attribute provisioning The standard Shibboleth approach to impose restrictions on attribute disclosure, consists of the identity provider configuring attribute filter policies. Therefore, decisions about the disclosure of personal information are taken by the identity provider on behalf of the user.

The proposed approach entrusts the user with the decision of which attributes to disclose. The introduction of this notion, renders Shibboleth Attribute Filter Policies obsolete and leads to a new question. Namely, how can the user be informed of the service provider's policy? The prototype stores this policy in the Shibboleth Metadata, similarly to the trusted set of trusted providers. This allows the identity broker to obtain user attributes on behalf of the service provider.

Secure credential storage Secure credential storage is implemented on the Giesecke \& Devrient Mobile Security Card 1.0 [1. The storage requirements differ, depending on the credential technology being used. The architecture in [22, for instance, relies on the tamperproofness of the card. Idemix however, can either be run entirely on 
the smartphone if speed is an important factor or with the master secret securely stored in the secure micro SD card.

\section{Evaluation}

This section evaluates to which extent the requirements from section 3.1 are fulfilled. A number of details and observations are discussed in 5.2. Paragraph 5.3 compares the merits and drawbacks of the proposed architecture to the two types of identity management it is inspired on. Finally, possible extensions are presented in 5.4

\subsection{Requirements review}

The identity broker is only responsible for user authentication and attribute provisioning. Storage of users' personal data is moved towards the claim providers, thereby reducing the identity broker's value and exposure as a target of attack. Additionally, some claim providers do not have to be online permanently, by which their issuance key is less susceptible to attack. Moreover, phishing attacks are less likely to be successful, due to the authentication feedback that is displayed by the user manager, in addition to the possibility to preconfigure the preferred identity broker on the user manager (see second paragraph of section 5.4 . Therefore, the architecture positively contributes to requirement G1.

Since users are no longer bound to the same party for personal data storage on one hand and authentication and attribute provisioning on the other, they can more easily switch identity brokers. The latter are now generic actors that support a variety of claim-based credential technologies and network-based authentication protocols, thereby giving users a broader choice and facilitating interoperability with service providers. Hence, requirement G2 is satisfied.

The identity broker remains a trusted party in the system. However, as a result of the properties of some claim-based credential technologies, the user's privacy is better protected. This is, for instance, the case in transactions where non-uniquely identifiable information is released. In addition, some authentications are anonymous, making it harder to monitor and link transactions and to compile profiles. Therefore, the architecture positively contributes to requirement U1.

The user agent shows information about the service provider and the requested user attributes during authentication. This allows the user to give his informed consent prior to the disclosure. This satisfies requirement $\mathrm{U} 2$.

Requirement U3 is trivially satisfied, since an identity broker in the architecture supports multiple claim-based credential technologies.

A typical Shibboleth identity provider maintains his own data storage, while claim providers in the proposed architecture endorse their provisioned data and thereby convince the identity broker of its correctness. As mentioned in sections 3.3 and 4 , service providers can specify a set of trusted claim providers. Therefore requirement SP1 is fulfilled.

Furthermore, the identity broker implements a variety of network-based authentication protocols, making it interoperable with many service providers. Therefore, requirement SP2 is fulfilled. 


\subsection{Discussion}

To evaluate differences in trust, we first identify the aspects by which these differences are influenced. Subsequently, we assess the actors to which the identified concerns apply. Finally, we link each of these concerns to the corresponding system actor. The resulting overview is presented in table 1. For the sake of brevity, the term "identity broker" is shortened to $I d B$. The same applies to $I d M$ (identity management), $I d P$ (identity provider) and $S P$ (service provider). To be accurate, we explain the link between the term issuer and identity provider. This section regards the issuer as an offline identity provider, typically in claim-based systems. However, some of the latter occasionally fetch attributes at authentication-time. Therefore, this division is not always clear-cut. This also explains why such systems might still have some degree of centralised attribute storage. To keep table 1 concise and to avoid unnecessary confusion, we consistently use the term issuer for claim-based systems. The $T$ in the table denotes a technology-dependent criterium. For instance, when considering user consent in PKI-based systems, the PIN-based authentication in the German eID enables user consent upon attribute disclosure. The Belgian eID, on the other hand, does not implement this measure. User consent in claim-based systems and in the proposed architecture, is often enforced by a combination of middleware and tamperproof hardware. Selective disclosure in network-based systems (if any), typically involves little user control. Improvements are provided by extensions such as uApprove (Shibboleth) and SimpleSAMLphp. Transactions in network-based systems are often linkable if no anonymous authentication or pseudonym change is supported.

The trust requirements are treated in the last three rows of the table. In networkbased systems, we observe strong a trust concentration in a single actor. Claim-based systems demand no trust in two out of three concerns. The proposed architecture has two important merits in this respect. Not only does it reduce the required user trust concerning profiling, transaction linking and impersonation. It also distributes the required trust over multiple actors.

An inherent advantage of the interoperability design criterium, is that the architecture allows identity brokers to use existing credential infrastructure to obtain required user attributes. This can prove benefical to the numerous eID architectures that have been deployed over the during the previous years.

\subsection{Comparison to existing systems}

\subsection{Extensions}

Prototype extensions The description of the second prototype setup in section 4.1 mentions that the QR code contains the ID of the session that is to be authenticated, which is a simplified configuration for demo purposes. In real-life setups, this configuration is prone to session hijacking (e.g. a bystander inconspicuously scanning the barcode at a distance). Therefore, the prototype uses a random authentication $i d$, of which the mapping to the corresponding session id is kept at the server side.

Section 4.1 also mentions the authentication step where the user is redirected to the Shibboleth Discovery Service, where he can select his preferred identity broker. Considerable anti-phishing protection can be put in place if this preference is pre- 


\begin{tabular}{|c|c|c|c|c|c|}
\hline & \multirow{2}{*}{$\begin{array}{l}\text { NETWORK- } \\
\text { BASED IDM }\end{array}$} & \multicolumn{2}{|c|}{ Claim-Based IdM } & \multicolumn{2}{|c|}{ HYBRID ARCH. } \\
\hline & & PKI & $\begin{array}{l}\text { Anon. } \\
\text { cred. }\end{array}$ & PKI & $\begin{array}{l}\text { Anon. } \\
\text { cred. }\end{array}$ \\
\hline User consent & Some systems & $T$ & Yes & $T$ & Yes \\
\hline $\begin{array}{l}\text { Selective disclo- } \\
\text { sure }\end{array}$ & Some systems & $T$ & Yes & $T$ & Yes \\
\hline $\begin{array}{l}\text { Attribute stor- } \\
\text { age }\end{array}$ & Centralised & $\begin{array}{l}\text { Credential }(+ \\
\text { centralised) }\end{array}$ & Credential & $\begin{array}{l}\text { Credential (+ } \\
\text { centralised) }\end{array}$ & Credentia \\
\hline $\begin{array}{l}\text { Linkability to- } \\
\text { wards IdP / IdB } \\
\text { (best case) }\end{array}$ & Yes & $T$ & No & No & No \\
\hline $\begin{array}{l}\text { Linkability } \\
\text { wards SP } \\
\text { case) }\end{array}$ & Often & $T$ & No & No & No \\
\hline \multicolumn{6}{|c|}{ Trust factors: concerns and actors } \\
\hline $\begin{array}{l}\text { SP concern: regis- } \\
\text { tration } \mathcal{E} \text { issuance }\end{array}$ & $\mathrm{IdP}$ & Issuer & & Claim provide & \\
\hline $\begin{array}{l}\text { SP concern: au- } \\
\text { thentication } \\
\text { prerequisite fulfill- } \\
\text { ment }\end{array}$ & IdP & - & & $\mathrm{IdB}$ & \\
\hline $\begin{array}{l}\text { User concern: no } \\
\text { profiling, transac- } \\
\text { tion linking, imper- } \\
\text { sonation }\end{array}$ & IdP & - & & $\mathrm{IdB}$ & \\
\hline
\end{tabular}

Table 1. Comparison to existing systems

configured instead having to select it each time. Little repetitive effort is required for this purpose, considering the generic nature of identity brokers.

In the prototype (section 4.1), the user agent receives the authentication challenge via a QR code, while the response is sent back through the device's Internet connection. Different alternatives are possible, however. For instance, an NFC or Bluetooth technology can be used for both the in- and outbound communication with the user agent. Taking advantage of the Internet connection of a different device, is also a possible option.

Enforcing trustworthy execution, is another issue to be taken into account. Browser hardening can be applied to prevent attacks where malware can trick the user into authenticating an illegitimate session or where malware piggybacks on the user's session. Trustworthiness can additionally be enforced on device running the user agent, to safeguard the integrity of the middleware.

Future plans for prototype implementation include the integration and evaluation of new credential technologies as well as authentication protocols other than Shibboleth. 
Different setups Interesting further research of the proposed approach, may include full integration of a trustworthy identity broker on the smartphone. This will most likely involve the application of tamperproof hardware.

The latter may also be applied at the identity broker's side, to further reduce required trust in this actor. Simultaneously, this would provide more privacy to otherwise less privacy-friendly credential technologies (e.g. X509 certificates).

\section{Conclusions}

This paper has presented a hybrid architecture that combines network-based and claim-based identity management architectures, leveraging multiple strengths of both and mitigating many of the weaknesses.

The approach offers improved trust, privacy and security properties, compared to several network-based systems, while claim-based identity management systems benefit from a greater extent of interoperability and standardisation, which are more prevalent in the network-based identity management ecosystem.

Additionally, a prototype demonstrates the feasibility of the proposed approach.

\section{Acknowledgements}

This work is made possible through funding from the MobCom project, by the Flemish agency for Innovation by Science and Technology (IWT). In this context, we also thankfully mention our joint collaboration with our fellow-researchers from the Steinbuch Centre for Computing, at the Karlsruhe Institute of Technology.

\section{References}

1. Mobile security card se 1.0. http://www.gd-sfs.com/the-mobile-security-card/mobilesecurity-card-se-1-0/.

2. Gergely Alpár, Jaap-Henk Hoepman, and Johanneke Siljee. The identity crisis. security, privacy and usability issues in identity management. CoRR, abs/1101.0427, 2011.

3. M. Alsaleh and C. Adams. Enhancing consumer privacy in the liberty alliance identity federation and web services frameworks. In Privacy Enhancing Technologies, pages 59-77, 2006.

4. Matthias Bauer and Martin M. H Meints. D3.1: Structured overview on prototypes and concepts of identity management systems. Technical report, FIDIS, 2005.

5. Jens Bender. The german eID-Card. presentation, September 2009.

6. Jens Bender, Dennis Kügler, Marian Margraf, and Ingo Naumann. Sicherheitsmechanismen für kontaktlose chips im deutschen elektronischen personalausweis - ein Überblick über sicherheitsmerkmale, risiken, und gegenmassnahmen. Datenschutz und Datensicherheit, 32(3):173-177, 2008.

7. Stefan A. Brands. Rethinking Public Key Infrastructures and Digital Certificates: Building in Privacy. MIT Press, Cambridge-London, 1 edition, August 2000.

8. Stefan A. Brands. The problem(s) with openid. webpage, August 2007.

9. J. Camenisch, I. Krontiris, A. Lehmann, G. Neven, C. Paquin, K. Rannenberg, and H. Zwingelberg. D2. 1 architecture for attribute-based credential Technologies-Version. Technical report, ABC4Trust Consortium, https://abc4trust.eu/index.php/pub/107d21architecturev1, December 2011.

10. Jan Camenisch and Els Van Herreweghen. Design and implementation of the idemix anonymous credential system. In ACM Conference on Computer and Communications Security, pages 21-30, 2002. 
11. David W. Chadwick. Federated identity management. In Alessandro Aldini, Gilles Barthe, and Roberto Gorrieri, editors, Foundations of Security Analysis and Design $V$, chapter Federated Identity Management, pages 96-120. Springer-Verlag, Berlin, Heidelberg, 2009.

12. Danny De Cock, Christopher Wolf, and Bart Preneel. The belgian electronic identity card (overview). In Sicherheit, pages 298-301, 2006.

13. B. Dodson, D. Sengupta, D. Boneh, and M. S Lam. Secure, consumer-friendly web authentication and payments with a phone. In Conference on Mobile Computing, Applications, and Services (MobiCASE'10), Santa Clara, CA, USA, 2010.

14. Orawiwattanakul et al. User-controlled privacy protection with attribute-filter mechanism for a federated sso environment using shibboleth. In Proceedings of $3 P G C I C 2010$, 3PGCIC '10, pages 243-249, Washington, DC, USA, 2010. IEEE Computer Society.

15. Sebastian Feld and Norbert Pohlmann. Security analysis of openid, followed by a reference implementation of an npa-based openid provider. In Norbert Pohlmann, Helmut Reimer, and Wolfgang Schneider, editors, ISSE 2010 Securing Electronic Business Processes, pages 13-25. Vieweg+Teubner, 2011.

16. Andreas Leicher, Andreas U. Schmidt, and Yogendra Shah. Smart OpenID: a smartcard-based OpenID protocol. In Proceedings of the 27th IFIP International Information Security and Privacy Conference, Heraklion, Crete, Greece, June 2012. Springer.

17. R. L. Morgan, Scott Cantor, Steven Carmody, Walter Hoehn, and Ken Klingenstein. Federated security : The shibboleth approach. EDUCAUSE Quarterly, 27(4), 2004.

18. Andreas Pashalidis and Chris Mitchell. A taxonomy of single sign-on systems. In Rei Safavi-Naini and Jennifer Seberry, editors, Information Security and Privacy, volume 2727 of Lecture Notes in Computer Science, pages 219-219. Springer Berlin / Heidelberg, 2003.

19. David Recordon and Drummond Reed. OpenID 2.0: a platform for user-centric identity management. In DIM '06: Proceedings of the second ACM workshop on Digital identity management, pages 11-16, New York, NY, USA, 2006. ACM.

20. International Telecommunication Union. Series y: Global information infrastructure, internet protocol aspects and next-generation networks: Next generation networks security. Recommendation ITU-T Y.2720, International Telecommunication Union, 2010 .

21. Kristof Verslype. Improving Privacy in Applications by Managing the Disclosed Personal Properties. PhD thesis, Katholieke Universiteit Leuven, March 2011.

22. Jan Vossaert, Jorn Lapon, Bart De Decker, and Vincent Naessens. User-centric identity management using trusted modules. In Proceedings of the rth European conference on Public key infrastructures, services and applications, EuroPKI'10, pages 155-170, Berlin, Heidelberg, 2011. Springer-Verlag.

23. Wikipedia. Id-wsf - wikipedia. webpage, May 2012.

24. Wikipedia. Shibboleth - wikipedia. webpage, May 2012. 